\title{
A NOTE ON 'BIG-MATCH'
}

\author{
JEAN-MICHEL COULOMB
}

\begin{abstract}
We present a very simple proof of the existence of the value
\end{abstract} for 'Big Match' first shown by Blackwell and Ferguson (1968).

\section{1. 'BIG-MATCH' GAME}

A 'Big-Match' game is a two person zero-sum repeated game with absorbing states, represented by the following $\{T, B\} \times\{1,2\}$ payoff matrix $C^{\star}:$

\begin{tabular}{c|c|c|}
\multicolumn{1}{c}{} & \multicolumn{1}{c}{2} \\
$\mathrm{~T}$ & \multicolumn{1}{c}{2} \\
\cline { 2 - 3 } $\mathrm{B}$ & $a_{1}^{\star}$ & $a_{2}^{\star}$ \\
\cline { 2 - 3 } & $b_{1}$ & $b_{2}$ \\
\cline { 2 - 3 } & &
\end{tabular}

where as usual a star $\star$ denotes an absorbing payoff. A play of the game is made of an infinite number of stages. At stage $k \geq 1$ player I (resp. II) selects one of the rows (resp. column $j_{k}$ ). Each player is told afterwards the choice of his opponent. One assumes perfect recall: both players remember the sequence of their own and their opponent's moves. The first stage where player I plays $\mathrm{T}$ is denoted by $\theta$. One defines the payoff $z_{k}$ at stage $k$ by:

$$
z_{k}= \begin{cases}b_{j_{k}} & \text { if } k<\theta \\ a_{j_{\theta}} & \text { otherwise. }\end{cases}
$$

Since one considers only the payoffs, the strategic choices of the players become irrelevant after stage $\theta$. Therefore a strategy $\sigma$ (resp. $\tau$ ) of player I (resp. II) specifies at stage $k$ a probability $\sigma_{k}\left(j_{1}, \ldots, j_{k-1}\right)\left(\sigma_{k}\right.$ for short) to play $\mathrm{T}$ (resp. $\tau_{k}\left(j_{1}, \ldots, j_{k-1}\right)$ to choose column 1$)$ when player II's previous moves are $j_{1}, \ldots, j_{k-1}$. The law of $\theta$ is induced by the couple of strategies $(\sigma, \tau)$. The expected average payoff up to stage $n$ under $(\sigma, \tau)$ is:

$$
\begin{aligned}
\gamma_{n}(\sigma, \tau) & =\mathbb{E}_{\sigma, \tau}\left(\frac{z_{1}+\ldots+z_{n}}{n}\right) \\
& =\mathbb{E}_{\tau}\left(\sum_{k=1}^{n} P_{\sigma}\left(\theta=k \mid j_{1}, \ldots, j_{k-1}\right)\left[\frac{(n-k+1) a_{j_{k}}+\sum_{h=1}^{k-1} b_{j_{h}}}{n}\right]\right. \\
& \left.+P_{\sigma}\left(\theta>n \mid j_{1}, \ldots, j_{n-1}\right) \frac{\sum_{h=1}^{n} b_{j_{h}}}{n}\right)
\end{aligned}
$$

URL address of the journal: http://www.emath.fr/ps/.

Received by the journal November 15, 1995. Revised June 5, 1996. Accepted for publication June 20, 1996.

(C) Société de Mathématiques Appliquées et Industrielles. Typeset by LATEX. 
Let us define:

$$
A_{n}(\sigma)=A_{n}\left(\sigma, j_{1}, \ldots, j_{n-1}\right)=\sum_{k=1}^{n} \frac{n-k+1}{n} P_{\sigma}\left(\theta=k \mid j_{1}, \ldots, j_{k-1}\right) a_{j_{k}} .
$$

Call $\Delta$ the set of probability distributions $y=\left(y_{1}, y_{2}\right)$ on $\{1,2\}$. Let $W=\min _{y \in \Delta} \max \left(y_{1} a_{1}+y_{2} a_{2}, y_{1} b_{1}+y_{2} b_{2}\right)$ be the value of the following matrix $C$ obtained from $C^{\star}$ by deleting the ' $\star$ 's:

$$
\begin{array}{|l|l|}
\hline a_{1} & a_{2} \\
\hline b_{1} & b_{2} \\
\hline
\end{array}
$$

One has the following result $([3])$ :

TheOREM 1. W is the value of the 'Big-Match' game.

Remark that player II can guarantee $W$ by playing i.i.d. an optimal strategy in the game $C$. In order to show that player I can guarantee $W$, we use a new approach based on two functions of a state variable.

REMARKS 1. Our definition of the value, the same as [5] and [6], is stronger than the one used in [3]. Here we do not associate a payoff to any play of the 'Big-Match' game. We show the $\epsilon$-optimality of the expected average payoff up to stage $n$ when $n$ becomes large, uniformly with respect to all strategies of player II. On the contrary, in [3] the payoff of a play is defined by $\limsup _{n \rightarrow \infty} \frac{z_{1}+\ldots+z_{n}}{n}$.

2. The functions that we use do not depend on the matrix $C^{\star}$ (at least under assuption 1 ).

In [3], one studies the case:

$$
C^{\star}=\begin{array}{|c|c|}
\hline 1^{\star} & 0^{\star} \\
\hline 0 & 1 \\
\hline
\end{array}
$$

\section{HEURISTIC}

We introduce a strategy which looks very much like the one introduced by Blackwell and Ferguson [3]. However our point of view seems to be quite different.

One starts from a simple remark: each time player I plans to choose $\mathrm{T}$ with a strictly positive probability, he takes the risk to get a bad i.e. strictly negative absorbing payoff. Furthermore he may accumulate these risks throughout the play. The new idea consists of monitoring at every stage the overall risk in the future that player $I$ is ready to take by using a function of a state variable. More precisely from stage $k$, the overall risk of player $\mathrm{I}$ is linked to $\sigma_{k}, a_{j_{k}}$ and the overall risk from stage $k+1$.

Player I uses two functions $f$ and $\tilde{\epsilon}$ of a parameter $x$ depending on player II's previous moves. The overall risk of player I (resp. the probability to play $\mathrm{T}$ ) is represented by $\tilde{\epsilon}(x)$ (resp. $f(x)$ ). These are given by the following ( $M$ is a large number to be fixed later):

$$
f(x)=\left\{\begin{array}{ll}
\frac{1}{1+(M+x)^{2}} & \text { if } M+x \geq 1 \\
\frac{1}{2} & \text { otherwise }
\end{array}\right\}
$$


and

$$
\tilde{\epsilon}(x)= \begin{cases}-\frac{1}{(M+x)} & \text { if } M+x \geq 1 \\ -1 & \text { otherwise }\end{cases}
$$

By straightforward computations we show that $f$ and $\epsilon$ satisfy (to some extend one can compare with [1] and [2]):

Lemma 2. For all $|\delta| \leq 1 / 2$ and for all $x$ we have:

$$
\delta f(x)+(1-f(x)) \tilde{\epsilon}(x-\delta) \geq \tilde{\epsilon}(x) .
$$

To find both functions, our heuristic is based on the functional inequation (2) which leads us to a set of differential equations. More precisely, up to second order one has:

$$
(1-f(x)) \tilde{\epsilon}(x-\delta) \simeq(1-f(x))\left(\tilde{\epsilon}(x)-\delta \tilde{\epsilon}^{\prime}(x)+\frac{\delta^{2}}{2} \tilde{\epsilon}^{\prime \prime}(x)\right) .
$$

To get rid of first degree terms in (2) one sets:

$$
(1-f(x)) \tilde{\epsilon}^{\prime}(x)=f(x) .
$$

Therefore one must verify:

$$
-f(x) \tilde{\epsilon}(x)+\frac{\delta^{2}}{2}(1-f(x)) \tilde{\epsilon}^{\prime \prime}(x) \geq 0 .
$$

It will hold if one sets the equality for $\delta=1$ i.e.:

$$
2 f(x) \tilde{\epsilon}(x)=(1-f(x)) \tilde{\epsilon}^{\prime \prime}(x) .
$$

Under the condition $0<f(x)<1$, one obtains:

$$
2 \tilde{\epsilon}(x) \tilde{\epsilon}^{\prime}(x)=\tilde{\epsilon}^{\prime \prime}(x) .
$$

\section{MAIN PROOF}

Given past moves $j_{1}, \ldots, j_{k-1}$ of player II, the expression $x_{k}=-\sum_{h=1}^{k-1} a_{j_{h}}$ $\left(x_{1}=0\right)$ will be used as state variable. The strategy $\sigma_{M}$ of player I consists of playing $\mathrm{T}$ at stage $k$ with probability $\sigma_{k}=f\left(x_{k}\right)$.

Proposition 3. For all $n \geq 1$ and for all strategy $\tau$ of player II, one has:

$$
\mathbb{E}_{\tau}\left(A_{n}\left(\sigma_{M}\right)\right) \geq-\frac{1}{M}
$$

Proposition 3 is a consequence of the following lemma since the function $\tilde{\epsilon}$ is always negative.

LEMMA 4. For all $n \geq 1$ and for all strategy $\tau$ of player II, one has:

$$
\mathbb{E}_{\tau}\left(A_{n}\left(\sigma_{M}\right)+\frac{1}{n} P_{\sigma_{M}}\left(\theta>n \mid j_{1}, \ldots, j_{n-1}\right) \tilde{\epsilon}\left(x_{n+1}\right)\right) \geq-\frac{1}{M} .
$$

Proof of the lemma:

For all $k=1, \ldots, n$, let us apply lemma 2 with $x=x_{k}$ and $\delta=a_{j_{k}}$ :

$$
\sigma_{k} a_{j_{k}}+\left(1-\sigma_{k}\right) \tilde{\epsilon}\left(x_{k+1}\right) \geq \tilde{\epsilon}\left(x_{k}\right) .
$$


Write $\epsilon_{k}=P_{\sigma_{M}}\left(\theta>k-1 \mid j_{1}, \ldots, j_{k-2}\right) \tilde{\epsilon}\left(x_{k}\right)$ and multiply both sides of $(4)$ by $\frac{n-k+1}{n} P_{\sigma_{M}}\left(\theta>k-1 \mid j_{1}, \ldots, j_{k-2}\right)$ to deduce that:

$$
\frac{n-k+1}{n} P_{\sigma_{M}}\left(\theta=k \mid j_{1}, \ldots, j_{k-1}\right) a_{j_{k}}+\frac{n-k}{n} \epsilon_{k+1} \geq \frac{n-k+1}{n} \epsilon_{k}
$$

since by negativity $\frac{n-k}{n} \epsilon_{k+1} \geq \frac{n-k+1}{n} \epsilon_{k+1}$. If one sums all these inequalities and remarks that $\epsilon_{1}=-1 / \stackrel{n}{M}$, then one obtains lemma 4.

This implies that the absorbing part of the payoff is 'controlled'. However it remains to study the non absorbing part of the payoff.

LeMma 5. For all $\epsilon>0$ and for all $k \geq 1$, if $\frac{1}{k} \sum_{h=1}^{k} b_{j_{k}} \leq-\epsilon$ then:

$$
P_{\sigma_{M}}\left(\theta>k \mid j_{1}, \ldots, j_{k-1}\right) \leq\left(\frac{M^{2}}{1+M^{2}}\right)^{[k \epsilon]}
$$

Proof:

Assume that:

$$
\frac{b_{j_{1}}+\ldots+b_{j_{k}}}{k}<-\epsilon .
$$

Remark that for all $k-k \epsilon \leq k^{\prime}<k$ one has:

$$
b_{j_{1}}+\ldots+b_{j_{k^{\prime}}}<0 \text {. }
$$

Since $W=0$, this implies that $-a_{j_{1}}-\ldots-a_{j_{k^{\prime}}}=x_{k^{\prime}+1} \leq 0$ and $\sigma_{k+1}=f\left(x_{k^{\prime}+1}\right) \geq \frac{1}{1+M^{2}}$. One then gets:

$$
P_{\sigma_{M}}\left(\theta>k \mid j_{1}, \ldots, j_{k-1}\right)=\prod_{h=1}^{k}\left(1-\sigma_{h}\right) \leq\left(\frac{M^{2}}{1+M^{2}}\right)^{[k \epsilon]}
$$

Finally we obtain:

Proposition 6. $\forall \epsilon>0, \exists N>0, \forall n \geq N, \forall \tau$ :

$$
\begin{aligned}
I_{\tau}\left(\sum_{k=1}^{n} \frac{k-1}{n} P_{\sigma_{M}}\left(\theta=k \mid j_{1}, \ldots, j_{k-1}\right) \frac{\sum_{h=1}^{k-1} b_{j_{h}}}{k-1}+\right. \\
\left.\quad P_{\sigma_{M}}\left(\theta>n \mid j_{1}, \ldots, j_{n-1}\right) \frac{\sum_{h=1}^{n} b_{j_{h}}}{n}\right) \geq-2 \epsilon .
\end{aligned}
$$

Proof:

Take $N_{1} \geq 1$ such that $\left(\frac{M^{2}}{1+M^{2}}\right)^{\left[N_{1} \epsilon\right]}<\epsilon / 2$ and $N_{2}$ such that $\frac{N_{1}}{N_{2}}<\epsilon / 2$. Let $\bar{k}$ be the first $k>N_{1}$ such that (5) holds. Therefore by lemma 5 , one has $P_{\sigma_{M}}\left(\theta>\bar{k} \mid j_{1}, \ldots, j_{\bar{k}-1}\right) \leq \frac{\epsilon}{2}$. Remark that for all $n \geq N_{2}$ :

$$
\begin{gathered}
\sum_{k=1}^{n} \frac{k-1}{n} P_{\sigma_{M}}\left(\theta=k \mid j_{1}, \ldots, j_{k-1}\right) \frac{\sum_{h=1}^{k-1} b_{j_{h}}}{k-1} \\
\quad+P_{\sigma_{M}}\left(\theta>n \mid j_{1}, \ldots, j_{n-1}\right) \frac{\sum_{h=1}^{n} b_{j_{h}}}{n}
\end{gathered}
$$




$$
\begin{aligned}
& \geq \quad-\frac{N_{1}}{N_{2}}-\epsilon P_{\sigma_{M}}\left(N_{1}+1<\theta \leq \bar{k} \mid j_{1}, \ldots, j_{\bar{k}-1}\right)-\frac{\epsilon}{2} \\
& \geq \quad-2 \epsilon .
\end{aligned}
$$

Propositions 3 and 6 show that player I can guarantee 0 since (1) is the sum of (3) and (6). This ends the proof of theorem 1.

\section{ACKNOWEDGEMENTS}

I would like to thank S. Sorin for his precious help and an anonymous referee for his very careful reading. The impulsion of this work originates from discussions with A. Neyman concerning my PhD dissertation.

\section{REFERENCES}

BlackWell D., (1969), Infinite $G_{\delta}$-Games with Imperfect Information, Applicationes Mathematicae, Hugo Steinhaus Jubilee Volume, X, 99-101.

BlackWfll D., (1989), Operator Solution of Infinite $G_{\delta^{-}}$Games of Imperfect Information, In Probability, Statistics and Mathematics. Papers in Honor of Samuel Karlin, ed. by T.W. Anderson, K.B. Athreya and D.L. Iglehart, Academic Press, 83-87.

Blackwell D. and Ferguson T.S., (1968), The Big Match, Ann. of Math. Stat, 39, 159-163.

Coulomb J.M., (1992), Repeated Games with Absorbing States and no Signals, Int. J. of Game Theory, 21, 161-174.

Kohlberg E., (1974), Repeated Games with Absorbing States, Ann. of Stat., 2, 724-738.

Mertens J-F. and Neyman A., (1981), Stochastic Games, Int. J. of Game Theory, 10, $53-56$.

UfR Segmi, Université Paris X, 200, avenue de la République, 92001 NanTERRE CEDEX. E-MAIL: coulomb@modalx.u-paris10.fr 